\title{
Effects of rosemary and green tea extracts on frozen surimi gels fortified with omega-3 fatty acids
}

\author{
Miriam Pérez-Mateos ${ }^{a}$, Tyre C. Lanier ${ }^{b}$ and Leon C. Boyd ${ }^{b}$
}

${ }^{\mathrm{a}}$ Instituto del Frío (CSIC), Madrid, Spain, and ${ }^{\mathrm{b}}$ North Carolina State University, Department of Food Science, Raleigh, NC 27695-7624, USA

(*)Corresponding author: Leon Boyd, North Carolina State University, Department of Food Science (E-mail: leon_boyd@ncsu.edu; (phone 919-513-2259, Fax 919-515-7124)

TITLE RUNNING HEAD Natural antioxidants in omega-3 surimi gels 


\begin{abstract}
Two different sources of omega-3 fatty acids (fish oil concentrate and menhaden oil) with or without the addition of natural antioxidants (rosemary and green tea) were incorporated into surimi gels at equivalent levels and examined for changes in sensory and physical properties and resistance to

20 oxidation during nine months of frozen storage. Gels with menhaden oil showed higher acceptance than gels with fish oil concentrate which displayed a fishy taste that was partially masked by natural antioxidants. Formation of volatile compounds was similar in all samples. Upon heating to form the gel, there was ca. 20-25\% decrease in the relative polyene index of the control containing no rosemary or green tea extract. Formulations with menhaden oil containing green tea and rosemary

25 were more stable immediately after cooking; however a slight pro-oxidant effect occurred during storage. Omega-3 fortified gels were whiter than gels with no added oil. Rosemary and green tea extracts increased yellowness $\left(b^{*}\right)$ and redness $\left(a^{*}\right)$, respectively. Strength increased in all formulations during frozen storage.
\end{abstract}

Key words: omega-3, surimi, lipid stability, natural antioxidants, frozen storage 


\section{INTRODUCTION}

35 Surimi-based products, such as shellfish analogue products, have proven to be tremendously popular due to their low cost and ease of preparation relative to natural shellfish meats. However, the surimi raw material has a very low content of desirable polyunsaturated omega-3 fatty acids (n3PUFA). As both eicosapentaenoic acid (EPA) and docosahexaenoic (DHA) have been shown to have a positive effect on reducing risk factors associated with several degenerative diseases, their

40 optimization in processed food products is important to improved human health. The Food and Drug Administration (FDA) recently approved the use of a "qualified health claim” for seafood products containing a significant amount of n-3 PUFA including EPA and DHA ${ }^{1}$.

There are three primary ways to enhance n-3 PUFA's in the diet; these being increased consumption of fatty fish species (i.e. salmon, tuna, mackerel), ingestion of tablets of encapsulated

45 fish oil, and fortification of n-3 PUFA into processed foods. As a formulated food, surimi seafoods derived from fish are a logical vehicle for increasing consumption of n-3 PUFA in the human diet. Supplementation of foods with n-3 PUFA using fish oil preparations in liquid and powdermicroencapsulated form has been practiced on a limited basis with varying levels of success ${ }^{2-4}$.

As n-3 PUFA are by nature highly unstable and subject to oxidation from various causes including

50 light, heat, metal, etc, their inclusion in formulated foods presents challenges for prevention of off flavors and aromas from detracting from product quality. Attainment of appropriate shelf life of n-3 PUFA-enriched products requires attention to the quality of the oil added, processing conditions, the inclusion of antioxidants and stabilizers, and appropriate packaging and storage conditions. Since synthetic antioxidants are restricted in use by amount and type under US food regulations whereas

55 many of the more effective synthetic antioxidants (e.g. TBHQ, BHT, BHA) are not acceptable and are often objectionable throughout the international food processing community, there is a trend 
toward the use of more natural antioxidants in conjunction with reduced synthetic antioxidants. As herbs and spices contain a variety of compounds, whose phenolic structures and antioxidant properties are similar to synthetic antioxidants, they may offer the opportunity to stabilize n-3

60 PUFA-enriched products while adding their own unique blend of special flavors and aromas ${ }^{5-6}$. Because of their effectiveness as antioxidants and flavoring agents, green tea and rosemary represent two of the most widely accepted groups of natural antioxidants currently added to formulated foods 7-11. However the stability of the oils and activity of antioxidants depend greatly on the particular food system (i.e. polarity and solubility of the antioxidant system in the food) ${ }^{8,12}$. The use of 65 multiple antioxidants having different modes of operation and different solubilities can often lead to synergism between antioxidants as has been shown to exist between rosemary extract and $\alpha$ tocopherol $^{13}$.

The objectives of this research were to fortify a crab analog product to an n-3 PUFA content equivalent to or greater than that present in natural salmon fillets (ca. $15 \mathrm{~g} \mathrm{~kg}^{-1}$ of flesh ${ }^{14}$ ) and to 70 determine its lipid stability and monitor the sensory properties in the presence of added natural antioxidants during a nine months frozen storage period. 


\section{MATERIALS AND METHODS}

Frozen surimi commercially prepared from Alaska pollock (Theragra chalcogramma) containing $40 \mathrm{~g} \mathrm{~kg}^{-1}$ sorbitol, $40 \mathrm{~g} \mathrm{~kg}^{-1}$ sucrose, $1.5 \mathrm{~g} \mathrm{~kg}^{-1}$ sodium tripolyphosphate and $1.5 \mathrm{~g} \mathrm{~kg}^{-1}$ tetrasodium pyrophosphate was obtained from Trident Seafoods Corporation (Seattle, WA). Frozen blocks of surimi (10 kg each) that had been held at $-20{ }^{\circ} \mathrm{C}$ were impact-shattered, portioned into $1 \mathrm{~kg}$ units, vacuum-packaged in oxygen impermeable bags (CN-590 cook-in material bags, Cryovac, Duncan,

80 SC), and stored at $-20{ }^{\circ} \mathrm{C}$ until needed. The moisture content of surimi was determined to be $76.4 \%$ 15 .

Preparation of surimi paste. Frozen surimi (400 g) was tempered at room temperature briefly, cubed, and chopped following the addition of sodium chloride (15 $\left.\mathrm{g} \mathrm{kg}^{-1} \mathrm{w} / \mathrm{w}\right)$ and other additives including $80 \mathrm{~g} \mathrm{~kg}^{-1}$ potato starch (Pencook 20 ${ }^{\mathrm{TM}}$, Penford Food Ingredients Co., Englewood, NJ); 20

$85 \mathrm{~g} \mathrm{~kg}^{-1}$ egg white powder (McWaldbaum, Wakefield, NE); $10 \mathrm{~g} \mathrm{~kg}^{-1}$ sorbitol (sorbitol 70/20, Roquette America, Inc., Keokuk, IA); $10 \mathrm{~g} \mathrm{~kg}^{-1}$ sucrose; $37 \mathrm{~g} \mathrm{~kg}^{-1}$ (w/w) of crab flavor and aroma (Activ International; Seattle, WA) and ice sufficient to achieve 75\% (w/w) moisture. This formulation closely resembles that of most commercial crab analog products being marketed.

In formulations containing added n-3 PUFA oils, oil replaced part of the added water by a 1:1 ratio

90 (w/w). Two commercial sources of n-3 PUFA were used: fish oil concentrate (20/50 TG ${ }^{\mathrm{TM}}$, Ocean Nutrition Canada, Ltd., Bedford, NS, Canada) and refined menhaden oil (Omega Pure ${ }^{\mathrm{TM}}$, Omega Protein, Reedsville, VA). The fish oil concentrate (Table 1) contained 89.5\% n-3 PUFA and mixed tocopherols. The refined menhaden oil contained 21.5\% n-3 PUFA and a combination of mixed tocopherols and tertiary butyl hydroxyquinone (TBHQ). The two oils were added at levels of $1.5 \%$

95 and 2.5\% n-3 PUFA (w/w) with adjustments made for differences in the amount of n-3 PUFA present in each oil: the amounts added for the fish oil concentrate for $1.5 \%$ and $2.5 \%$ n-3 PUFA 
were $16.8 \mathrm{~g} \mathrm{~kg}^{-1}$ and $27.9 \mathrm{~g} \mathrm{~kg}^{-1}$, respectively; whereas the menhaden added for $1.5 \%$ and $2.5 \% \mathrm{n}-3$ PUFA oil was $69.8 \mathrm{~g} \mathrm{~kg}^{-1}$ and $116.2 \mathrm{~g} \mathrm{~kg}^{-1}$, respectively).

The antioxidants were added directly to the lipid phase before its addition to surimi. Green tea 100 extract $\left(0.6 \mathrm{~g} \mathrm{~kg}^{-1}\right.$ of total product) containing $85 \%$ pure polyphenols (i.e. primarily catechins; Templar Food Products, New Providence, NY) and rosemary (0.75 $\mathrm{g} \mathrm{kg}^{-1}$ of total product) (Herbalox $^{\mathrm{TM}}$ seasoning Type HT-O, Kalsec Kalamazoo, MI, USA) were used at the recommended level to avoid bitter taste.

Surimi pastes were extruded into stainless steel tubes $(22.7 \mathrm{~cm}$ length, $1.9 \mathrm{~cm}$ i.d.) and cooked as

105 described by Perez-Mateos et al. ${ }^{16}$. The gels were frozen at $-40{ }^{\circ} \mathrm{C}$ in a freezer for 5 hours, and kept in frozen storage at $-18{ }^{\circ} \mathrm{C}$ for 9 months. Analyses were done every 3 months. Samples were completely thawed at $4^{\circ} \mathrm{C}$ overnight before analyses.

Sensory evaluation. A six-member trained sensory panel ${ }^{17,18}$ performed descriptive sensory analyses. Aroma, flavor, and after-taste notes that included sweet-corn, crab, fishy, briny, herbal, 110 salty and astringent were determined using a fourteen-point universal scale. The points of anchor on the scale were as follows: not detectable (0), threshold (1-5), slight (6-9), moderate (10-13), and strong (14). The panel had extensive experience evaluating flavor changes in fish gels and was retrained in this study to score crab product attributes with an emphasis on recognizing fishy taste and aroma as these are the two negative sensory attributes most often associated with the addition of 115 n-3 PUFA. Control gels containing no added oil and stored at $-50{ }^{\circ} \mathrm{C}$ were used for reference. Each sensory property was expressed as the average of the data from all panelists.

Lipid Stability. Production of lipid oxidation volatiles was determined by the manual solid phase microextraction (SPME) technique for low molecular weight oxidation compounds ${ }^{19}$. A SPME extractor containing a $75 \mu \mathrm{m}$ filter of Carboxen ${ }^{\mathrm{TM}}$ polydimethylsiloxane (Supelco, Bellefonte, PA) 
120 was used. Two g of chopped gel were placed in a $12 \mathrm{~mL}$ vial, flushed with nitrogen, closed with a headspace clamp, and kept in frozen storage at $-18^{\circ} \mathrm{C}$. Headspace was sampled initially at 0 months and at the conclusion of the 9 months storage. Oxidation volatiles present in surimi gels were extracted onto SPME fibers by heating vials at $60{ }^{\circ} \mathrm{C}$ for 15 min with the manual SPME apparatus in the injection mode. Desorption of volatiles from the fiber onto a GC column occurred by heating the

125 SPME apparatus in GC injection port for $2 \mathrm{~min}$ at $180^{\circ} \mathrm{C}$ with a carrier gas flow rate of $2.0 \mathrm{~mL} / \mathrm{min}$. An HP-5 capillary column (30 m x 0.25 mm i.d., $0.25 \mu \mathrm{m}$ film thickness, Hewlett Packard, Palo Alto, CA) was used for separation. Volatiles were identified by comparison to authentic standards followed by confirmation with a GC-mass spectrometry system consisting of a HP 5890 II GC and HP 5972 mass selective detector. The GC oven temperature was cryogenically cooled and 130 programmed from $-20{ }^{\circ} \mathrm{C}$ to $160^{\circ} \mathrm{C}$ at $15{ }^{\circ} \mathrm{C} / \mathrm{min}$ with initial and final hold times of 4.5 and 1 min, respectively. The oven temperature was programmed for an increase of $40^{\circ} \mathrm{C} / \mathrm{min}$ from $160{ }^{\circ} \mathrm{C}$ to $220{ }^{\circ} \mathrm{C}$ and a final hold time of $1.5 \mathrm{~min}$. The carrier gas was electronically controlled at a linear velocity of $1.5 \mathrm{~mL} / \mathrm{min}$. The ionization voltage for the mass spectrometer was $70 \mathrm{eV}$. The mass scanning range was 35-350 kDa.

135 To determine the fatty acid composition of each oil and of n-3 PUFA-enriched gels, total crude lipids were extracted by a modification of the Bligh and Dyer ${ }^{20}$ procedure in which $0.001 \mathrm{~g} / \mathrm{L}$ of BHT was added to a chloroform / methanol solvent system (1:2), followed by extraction, cleanup, and subsequent drying of lipids. The fatty acid methyl esters (FAME) were prepared by the addition of $2 \mathrm{~mL}$ boron trifluoride-methanol per $25 \mathrm{mg}$ of lipid. Crude FAME were washed with distilled 140 water, extracted twice with $2 \mathrm{~mL}$ of hexane, and dried under nitrogen at $35^{\circ} \mathrm{C}^{21}$. Crude FAME were purified by thin layer chromatography. Bands of FAME were compared to FAME standards, scraped from TLC plates, and eluted with a petroleum ether/diethyl ether (95:5) solvent system, followed by drying under nitrogen. Purified FAME were re-dissolved in iso-octane for injection. The FAME 
were analyzed on a GC (HP model 5890A), equipped with a flame ionization detector. The GC 145 contained a DB 225, $30 \mathrm{~m}$ x $0.25 \mathrm{~mm}$ fused silica column (J\&W Scientific Co, Folsom, CA) that was temperature programmed from $140{ }^{\circ} \mathrm{C}$ to $200{ }^{\circ} \mathrm{C}$ at a rate of $1.7^{\circ} \mathrm{C} / \mathrm{min}$ with both injector and detector temperatures set at $260{ }^{\circ} \mathrm{C}$. Identification of FAME was based on comparison of retention times to methyl ester standards (462 and 68D, NuChek Prep, Elysian, MN) using C21:0 as the internal standard. Absolute response factors were calculated for each identified fatty acid peak using

150 the normalization technique described by Sampugna et al ${ }^{22}$. Fatty acid composition was expressed as weight percent of total FAME.

Polyene index (PI: ratio of polyunsaturated (PUFA) to saturated (SAT) fatty acids) was calculated from the gas chromatographic data. As DHA represents the most highly unsaturated fatty acid, and therefore, the most labile fatty acid present in fish oils, the loss of DHA relative to palmitic acid

155 (C16:0) was used to calculate PI in the present study ${ }^{23-24}$. Data are reported as percentages of the PI of the stock oil.

Color Analysis of Gels. Color differences between treated gels and the control were determined by reflectance on a Chroma Meter CR 300 (Minolta Camera Co., Ltd., Osaka, Japan) using the CIE Lab scale $\left(\mathrm{D} 65 / 10^{\circ}\right)$, where $\mathrm{L}^{*}$ (black 0 to light 100 ), a* (red 60 to green -60 ) and $\mathrm{b}^{*}$ (yellow 60 to 160 blue -60) were used to measure lightness, redness, and yellowness. Whiteness Index was defined as: $100-\left[\left(100-\mathrm{L}^{*}\right)^{2}+\mathrm{a}^{2}+\mathrm{b}^{*^{2}}\right]^{1 / 2}$. Results are expressed as the average of six samples per formulation.

Gel Fracture Properties. Gel samples were tempered to ambient temperature $\left(20{ }^{\circ} \mathrm{C}\right)$ prior to analysis. Gels were cut into 29-mm long cylinders, each end of which was glued to plastic disks using cyanoacrylate glue. The cylinders were ground on a Precision Machine (Model GC-TG92 US, 165 Gel Consultants, Inc., Raleigh, NC) and formed into capstan shapes with a center diameter of 10 mm. Gels were vertically mounted and twisted to fracture at $2.5 \mathrm{rpm}$ on a Hamann Torsion 
Gelometer to determine stress (strength) and strain (deformability) at fracture as described by Diehl et al $^{25}$.

Statistical Analysis. One-way analysis of variance (ANOVA) was carried out using the SPSS 170 system (SPSS Inc., Chicago, Illinois). For laboratory samples, significant differences between pairs were determined by the Bonferroni test. For sensory tests, significant difference between means were resolved with confidence intervals using non parametric statistics by the Kruskal-Wallis test. Level of significance was set at $\mathrm{p}<0.05$.

Sensory testing. Figure 1 shows the profile of the descriptive sensory attributes of a typical formulation of surimi gels containing no added oil. The attributes typical of crab flavoring (mainly sweet corn and crab aroma) decreased significantly $(\mathrm{p}<0.05)$ during frozen storage showing decreases of $53 \%$ for sweet-corn aroma and $61 \%$ for crab aroma.

180 The addition of fish oil in food fortification is limited by its fishy flavor and high capacity of n-3 PUFA to oxidize resulting in an undesirable taste and odor and subsequent reduction in the shelf life of products. For this reason, palatability is one of the main characteristics limiting the level of fortification with n-3 PUFA. In this study, $2.5 \%$ n-3 PUFA was the highest level that was acceptable to the sensory panel (taste and texture) whereas levels of $3 \%$ were deemed unacceptable

185 (data not shown). Samples containing $1.5 \%$ n-3 PUFA from fish oil concentrate (Fig.2) acquired a clear fishy taste (i.e. aroma, flavor and aftertaste) that significantly increased with storage time reaching scores of 5.3 for fishy aroma and 5.7 for fishy flavor in comparison to scores of 3.8 and 4.0 for gels with no oil added (Fig. 1). The addition of strong flavored antioxidants (i.e. rosemary and green tea) was effective in decreasing the moderate fishy flavors and aromas caused by the addition 190 of the concentrated fish oil as noted by lower fishy aroma (3.8) and flavor (3.9) scores. 
The intensity of crab flavor and aroma in the gels with fish oil concentrate at a n-3 PUFA of $1.5 \%$ (Figure 2) was lower than in gels with no oil added (Figure 1) but was higher when antioxidants were present in the formulation. Even though herbal notes from the addition of natural antioxidants, such as tea and rosemary extract, are often limiting factors preventing their use, in this particular 195 study, the strong flavor and aroma of the crab flavoring was effective in masking herbal notes as well as suppressing both fishy taste and aroma. Manley et al. ${ }^{26}$ reported that the addition of oil decreases the vapor pressure of many flavor components due to their affinity toward the oil. This also may be the reason herbal notes did not increase in samples with antioxidants added.

A similar pattern of changes in sensory attributes was seen for gels containing fish oil concentrate 200 at the higher level (2.5\% of n-3 PUFA) (Fig.3) but with higher scores in the fishy attributes. Overall sensory attributes were improved significantly by rosemary or green tea extract addition; thus slowing and masking the off flavor effects of the higher fish oil concentrate level.

Formulations containing menhaden oil with and without rosemary and green tea extracts (Fig.4 and 5) did not have increased fishy flavor and aromas as compared to the control gel with no oil 205 added (Fig.1). Mean fishy flavor and aroma for formulations containing menhaden oil added without antioxidants were only 4.1 and 2.9, respectively, compared to scores of 5.3 and 5.7 observed in the fish oil concentrate-containing samples with no extracts. Additionally, over the frozen storage period, fishy aroma and flavor increased in samples containing fish oil concentrate (Fig 2-3) whereas in samples containing menhaden oil, fishy aroma and flavor remained constant. Crab flavor scores

210 (Fig 4-5) for the formulations containing menhaden oil did not change during the 9-months study compared to formulations containing fish oil concentrate (Fig 2-3), which showed a decrease in crab flavor over storage time.

Butler and Larick ${ }^{27}$ reported that the use of rosemary improved the sensory characteristics and oxidative stability of heat-induced beef gels. Karpinska et al. ${ }^{28}$ found that rosemary extract decreased 
215 rancid taste and aroma of meat dishes and the rate of undesirable changes was reduced during frozen storage. Similarly Stoick et al. ${ }^{10}$ found a linear concentration effect of rosemary improving sensory scores of beef steaks indicating effective antioxidant activity during frozen storage. A study conducted by Boyd et al. ${ }^{11}$ also showed improved sensory scores and decreased oxidation products in cooked trout flakes with the addition of rosemary extract.

\section{$220 \quad$ Lipid stability}

The formation of volatiles (aldehydes and alcohols) is highly correlated with the loss of DHA during storage of fish oil ${ }^{29}$. The refining process in fish oil production removes almost all compounds related to fishy odor; however during storage, undesirable volatiles can be generated including pentylfuran creating and fishy odor and selected aldehydes having green and beany odors.

225 Additionally the generation of threshhold levels of ketones can produce metallic off-flavors such as decatrienal and heptenal that impart fishy odors ${ }^{30}$.

In our study, sensory analysis showed differences between the formulations; however these differences were not detected by SPME GC-MS, in that similar formation of volatile compounds was detected in all samples (data not shown). No significant differences due to type of oil or

230 antioxidant added were evident in any of the formulations using this methodology. Among 10-20 peaks detected, the main compounds positively identified were acetaldehyde, ethanol, dimethyl sulfide, ethyl acetate, butanediol and hexanoic acid. After 3 months of frozen storage, hexanal was detected in most of the gels whereas pentanal was found in a few gels; however, no significant differences in volatiles were detected between any of the formulations.

235 Although other volatile analysis techniques are available, SPME was chosen in this study due to its precision. Comparison of the SPME technique to Purge and Trap (PT) reveal differences in both the level of detection and precision. For example, SPME is reported to have a detection limit of parts 
per thousand and a precision of 1-12 \% relative standard deviation (RSD) whereas PT has a lower limit of detection of parts per billion (ppb) but a precision of $1-30 \%{ }^{31}$. PT is also typically done at 240 higher temperatures that can generate sample degradation, thus contributing to the background noise often preventing distinction between artifacts generated from sample preparation vs. oxidation actually occurring in the sample. More recent studies by Mindrup ${ }^{32}$ showed detection limits of SPME down to ppb when applied to off-flavor volatiles from light abused $2 \%$ milk.

Studies have shown that the production of volatiles is sensitive to the degree of unsaturation of the 245 oil. Pentanal and hexanal are the two main volatiles associated with n-3 PUFA and omega-6 (n-6) oxidation, respectively ${ }^{21}$. Yu et al. ${ }^{33}$ and Lai et al. ${ }^{34}$ reported that hexanal decreased during storage when rosemary extract was present.

Results from our study suggest that since the oils used contained commercial and natural antioxidants, their presence may have had an impact toward stabilizing oils during frozen storage 250 resulting in no significant differences in volatiles produced.

One other factor that may have contributed to lack of differences observed between stored formulations by the SPME technique is that the sampling time may not have corresponded to the observed oxidation detected by the sensory panel. Samples for SPME analyses were done at the beginning of the study ( 0 months) and at the end of the study ( 9 months). Closer examination of 255 sensory data (Fig. 1-5) shows that maximum detection of fishy flavor and aroma occurred between the $3^{\text {rd }}$ and $6^{\text {th }}$ months. Therefore, a number of factors including use of the SPME technique, effectiveness of the antioxidant systems in the oils and sampling time as well as the efficiency of the processing systems used to produce the n-3 PUFA fish oils, may all have contributed to the lack of differences observed using the SPME technique.

260 For samples containing fish oil concentrate or menhaden oil (Fig.6), the heating process used to form gels resulted in a 20-25 \% decrease in the polyene index (PI) ratio compared with the stock oil. 
A lower PI ratio for oils extracted from cooked surimi gels was expected since most unsaturated fatty acids are heat-labile. During subsequent frozen storage, the gels containing appeared to be quite stable with no reductions in n-3 PUFA composition observed.

265 An antioxidant effect (about $10 \%$ greater PI ratio) of green tea and rosemary was noted in formulations containing menhaden oil immediately after gel preparation, but was not observed toward the end of the study. This limited effect of the antioxidants during storage might be explained by the mechanism of the individual antioxidants in the system. Previous studies ${ }^{35}$ show that rosemary's effectiveness as a primary antioxidant maybe due to its reaction with initial free radicals

270 to form stable products.. Therefore it is likely that the antioxidants used in the present study were effective only at limiting the free radical production during cooking but not be very effective for long-term prevention of oxidation. Additionally, the addition of rosemary and green tea could produce a pro-oxidant effect after storage resulting in the reduced stability of the oil ${ }^{36}$.

\section{Color}

275 Figure 7 shows how the addition of both oils significantly increased the whiteness of gels. This increased whiteness was mainly due to the increase in lightness $\left(\mathrm{L}^{*}\right)$ attributed to the light scattering effect that results from the emulsion created when oil is mixed with surimi proteins and water, though there was also some changes in redness $\left(\mathrm{a}^{*}\right)$ and yellowness $\left(\mathrm{b}^{*}\right)$ due to the level of oil added (Table 2 and 3). There was no correlation between whiteness and the percentage of oil added,

280 however, since formulations containing fish oil concentrate (with up about $3 \%$ oil) had the same or higher whiteness as formulations containing menhaden oil (with up about $12 \%$ oil).

The antioxidants themselves caused color changes in the surimi gels (Table 2 and 3). Rosemary significantly increased yellowness (b*) in the gels; whereas green tea extract significantly increased redness $\left(a^{*}\right)$ in all the samples. However, no significant changes during frozen storage were 285 observed (data not shown). 


\section{Rheological changes}

At the beginning of storage, the texture of all gels was similar across different formulations with and without addition of oils or antioxidants (Fig.8). However, a similar study carried out by the investigators for gels kept under chilled storage ${ }^{16}$ showed higher stress in surimi gels containing

290 menhaden oil. This difference could be due to the detrimental effect of the freezing process on the texture of the gels.

After 3 months of storage, stress (strength) decreased significantly for all formulations, but then significantly increased during the following months. These changes were concurrent with observed syneresis that occurred during each of the thawing procedures in preparing samples for texture

295 measurements. At the end of storage, formulations containing menhaden oil had the highest shear stress which can probably be attributed to the higher amount of oil added in this formulation (up to about $12 \%$ ) in comparison with about $3 \%$ in the formulation with fish oil concentrate. The menhaden oil had a lower content of n-3 PUFA and therefore greater amounts were added in order to obtain an equivalent level of n-3 PUFA present in the fish oil concentrate formulation (Table 1). Lee

300 and Abdollahi ${ }^{37}$ suggested that the addition of oil to fish protein gels prevents sponge-like texture development during extended frozen storage by improving freeze-thaw stability.

Shear strain values (Figure 9) showed no significant difference between formulations with or without the addition of oil or antioxidant at the beginning of storage. However, during frozen storage, there was a trend toward decreasing shear strain across all the formulation. Similar studies

305 by Butler and Larick ${ }^{27}$ examining the behavior of shear stress and shear strain in heat-induced lowfat beef gels in which they demonstrated that neither parameter was affected by the addition of antioxidants in the formulations. Recently, however Pérez-Mateos et al. ${ }^{38}$ reported that the addition of rosemary extract and n-3 PUFA to mackerel mince gels had a greater impact on breaking force than breaking deformation. 


\section{CONCLUSIONS}

The herbal sensory attributes of rosemary and green tea can be masked by the fishy flavor and aromas of fish oil concentrate added to these formulations. However, the addition of a moderate to

315 strong flavored additive, such as those present in crab flavoring may have a positive effect by partially masking both the herbal flavor of natural antioxidants and fishy off-flavors resulting from the enrichment of formulated products with n-3 PUFA. In this study, all the samples containing added fish oil concentrate and menhaden oil were fairly stable during frozen storage. While much of the observed stability of the lipids might be attributed to the combined affects of stabilizers already

320 added to the commercial oils during industrial processing as well as newer processes used to produce superior fish oils, this study also showed that the addition of natural antioxidants may add to both the masking of fishy flavor and aromas while preventing the occurrence of measurable oxidation products. From the results obtained in this study, fortified crab-flavored surimi seafood products are an adequate vehicle to increase consumption of n-3 PUFA in formulated meat products,

325 including seafoods. Further studies might also investigate whether higher levels of rosemary and green tea extracts could more effectively mask the fishy flavor and aroma associated with the addition of the fish oil concentrate, since the fish oil concentrate adds fewer calories to achieve the same level of n-3 PUFA, compared to menhaden oil. However, the fishy aroma after-taste associated with it must be overcome in order to recommend its use. 


\section{ACKNOWLEDGMENT}

Paper number FSR-04-16 of the journal series of the Department of Food Science, NC State University. The use of trade names does not imply endorsement, nor criticism of similar ones not

335 mentioned. This research was funded by the North Carolina Agricultural Research Service of the College of Agricultural and Life Science, the University of NC Sea Grant College Program and the National Fisheries Institute. Dr. M. Pérez-Mateos is grateful to the Spanish Ministry of Education, Culture and Sport (MECD) and the Fulbright Program for the sponsorship of her postdoctoral fellowship. 


\section{REFERENCES}

1. US Food and Drug Administration, FDA Announces Qualified Health Claims for Omega-3 Fatty Acids. http://www.fda.gov/bbs/topics/news/2004/NEW01115.html [accessed 8 September 2004].

345 2. Metcalf RG, James MJ, Mantzioris E and Cleland LG, A practical approach to increasing intakes of n-3 polyunsaturated fatty acids: use of novel foods enriched with n-3 fats. European J Clinical Nutrition 57(12):1605-1612 (2003).

3. Park Y, Kelleher SD, McClements DJand Decker EA, Incorporation and stabilization of omega-3 fatty acids in surimi made from cod, Gadus morhua. J Agric Food Chem 52(3):597-601 (2004).

4. Kolanowski W Laufenberg G Kunz B, Fish oil stabilisation by microencapsulation with modified cellulose. Intern J Food Sci Nutrition 55 (4):333-343 (2004).

5. Nakatani N, Phenolic antioxidants from herbs and spices. BioFactors 13:141-146 (2000).

6. Wanasundara UN and Shahidi F, Stabilization of marine oils with flavonoids. J Food Lipids 5 (3):183-196 (1998).

7. He YH and Shahidi F, Antioxidant activity of green tea and its catechins in a fish meat model system. J Agri Food Chem. 45(11):4262-4266 (1997).

8. Huang SW and Frankel EN, Antioxidant activity of tea catechins in different lipid systems. J Agri Food Chem. 45(8):3033-3038 (1997). 
9. Barbut S, Josephson DB and Maurer AJ, Antioxidant properties of rosemary oleoresin in turkey sausage. J Food Sci 50:1356-1363 (1985).

10. Stoick SM, Gray JI, Booren AM and Buckley DJ, Oxidative stability of restructured beef steaks processed with oleoresin rosemary, tertiary butylhydroquinone, and sodium tripolyphosphate. J Food Sci 56:597-600 (1991).

11. Boyd LC, Green DP, Giesbrecht FB and King MF, Inhibition of oxidative rancidity in frozen cooked fish flakes by tert-butylhydroquinone and rosemary extract. J Sci Food Agric 61:87-93 (1993).

12. Frankel EN, Huang SW, Aeschbach R and Prior E, Antioxidant activity of a rosemary extract and its constituents, carnosic acid, carnosol, and rosmarinic acid, in bulk oil and oilin-water emulsion. J Agri Food Chem 44(1):131-135 (1996).

13. Wada $\mathrm{S}$ and Fang $\mathrm{X}$, The synergistic antioxidant effect of rosemary extract and $\alpha$ tocopherol in sardine oil model system and frozen-crushed fish meat. J Food Process Preserv 16:263-274 (1992).

14. Bell JG, McEvoy J, Webster JL, McGhee F, Millar RM and Sargent JR, Flesh lipid and carotenoid composition of Scottish farmed Atlantic salmon (Salmo salar). J Agric Food Chem 46:119-127 (1998).

15. AOAC. Official Methods of Analysis, 14 ed. Assoc. of Official Analytical Chemists, Arlington, VA. (1984).

16. Pérez-Mateos M, Boyd L and Lanier T, Stability of omega-3 fatty acids in fortified surimi seafoods during chilled storage. J Agri Food Chem 52(26):7944-7949 (2004). 
17. Stone J, Siedel J, Oliver S and Woosley A, Sensory evaluation by quantitative descriptive analysis. Food Technol 28:24-34 (1974).

18. Henry LK, Boyd LC and Green DP, The effects of cryoprotectants on the sensory properties of frozen blue crab (Callinectes sapidus) Meat. J Sci Food Agric 69:21-26 (1995).

19. Brunton NP, Cronin DA, Monahan FJ and Durcan R, A comparison of solid-phase microextraction (SPME) fibers for measurement of hexanal and pentanal in cooked turkey. Food Chem 68:339-345 (2000).

20. Bligh EG and Dyer WJ, A rapid method of total lipid extraction and purification. Can $J$ Biochem Physiol 37:911-914 (1959).

21. Metcalfe LD and Schmitz AA, The rapid preparation of fatty acid esters for gas chromatographic analysis. Anal Chem 33:363-368 (1961).

22. Sampugna J, Pallansch LA, Enig MG and Keeney M, Rapid analysis of trans fatty acids on SP-2340 capillary columns. J Chromatogr 249:245-255 (1982).

23. Boyd LC, King MC and Sheldon BA, Rapid method for determining the oxidation of n-3 fatty acids. J Am Oil Chem Soc 69:325-330 (1992).

24. King MF, Boyd LC and Sheldon BW, Antioxidant properties of individual phospholipids in a salmon oil model system. J Am Oil Chem Soc 69(6):545-551 (1992).

25. Diehl KC, Hamann DD and Whitfield JK, Structural failure in selected raw fruits and vegetables. J Text Studies 10 (4):371-400 (1980). 
26. Manley C Mankoo A Dubosc V, Surimi Seafood Flavor, in Surimi and Surimi Seafood, Ed by Park JW. CRC Press, (2nd Ed), Boca Raton FL, Chapter 14, pp.709-748 (2005).

27. Butler AJ and Larick DK, Effect of antioxidants on the sensory characteristics and storage stability of aseptically processed low-fat beef gels. Meat Sci 35:355-369 (1993).

28. Karpinska M, Borowski J and Danowska-Oziewicz M, Antioxidative activity of rosemary extract in lipid fraction of minced meat balls during storage in a freezer. Nahrung 44(1):3841 (2000).

29. Lee H, Kizito SA, Weese SJ, Craig-Schmidt MC, Lee Y, Wei CI and An H, Analysis of headspace volatile and oxidized volatile compounds in DHA-enriched fish oil on accelerated oxidative storage. J Food Sci 68 (7):2169-2177 (2003).

30. Lin CF, Flavor chemistry of fish oil, in Lipids in food flavors, Ed by Ho CT, Hartman TG. ACS symposium series, Chapter 5, pp.208-232 (1994).

31. Shirey RE, Wooley CL and Mindrup RF, Solventless extraction of flavors and other food components. Food Testing \& Analysis 39-42 (1995).

32. Mindrup RF, Recent applications of SPME for monitoring flavor components in foods. Food Testing \& Analysis 17-19 (2000).

33. Yu L, Scanlin L, Wilson J, Schmidt G, Rosemary extracts as inhibitors of lipid oxidation and color change in cooked turkey products during refrigerated storage. J Food Sci 67(2):582-585 (2002). 
34. Lai SM, Gray JI, Smith DM, Booren AM, Crackel RL and Gill JL, Assessment of offflavor development in restructured chicken nuggets using hexanal and TBARS measurements and sensory evaluation. J Sci Food Agric 67:447-452 (1995).

35. Langourieux S and Escher FE, Sulfurous off-flavor formation and lipid oxidation in heatsterilized meat in trays. J Food Sci 63(4):716-720 (1998).

425 36. Boyd LC, Application of natural antioxidants in stabilizing polyunsaturated fatty acids I model systems and foods, in Omega-3 fatty acids Chemistry, Nutrition, and Health Effects, Ed by Shahidi F and Finley J. ACS Symposium Series 788, Chapter 20, pp.258-279 (2001).

37. Lee CM, Abdollahi A, Effect of hardness of plastic fat on structure and material properties of fish-protein gels. J Food Sci 46 (6):1755-1759 (1981).

38. Pérez-Mateos M, Gómez-Guillén MC, Hurtado JL, Solas T and Montero P, The effect of rosemary extract and omega-3 unsaturated fatty acids on the properties of gels made from the flesh of mackerel (Scomber scombrus) by high pressure and heat formulations. Food Chemistry 79 (1):1-8 (2002). 
Table 1. Summary of fatty acids compositions of fish oil concentrate and menhaden oil as percentage composition of total fatty acids identified.

\begin{tabular}{lcc}
\hline $\begin{array}{l}\text { Major groups of fatty } \\
\text { acids }\end{array}$ & Fish Oil Concentrate & Menhaden Oil \\
& 2.0 & 41.5 \\
\hline Saturates & 6.1 & 34.0 \\
Monoenes $^{\text {PUFA }}{ }^{\text {(a) }}$ (n-3 PUFA $\left.{ }^{(b)}\right)$ & 91.9 (89.5) & 24.5 (21.5) \\
\hline PUFA $=$ total polyunsaturated fatty acids; (b) total n-3 polyunsaturated fatty acids
\end{tabular}


Table 2. Color and standard deviation of surimi gels with fish oil concentrate and antioxidants addition: redness $\left(\mathrm{a}^{*}\right)$ and yellowness $\left(\mathrm{b}^{*}\right)$ at the beginning of storage time.

\begin{tabular}{ccccccc}
\hline Fish oil & \multicolumn{2}{c}{ No antioxidant } & \multicolumn{2}{c}{ Rosemary } & \multicolumn{2}{c}{ Green tea } \\
concentrate & $\mathrm{a}^{*}$ & $\mathrm{~b} *$ & $\mathrm{a}^{*}$ & $\mathrm{~b}^{*}$ & $\mathrm{a}^{*}$ & $\mathrm{~b}^{*}$ \\
\hline $1.5 \%$ & -1.84 & 5.75 & -2.02 & 7.93 & -0.06 & 5.51 \\
& $\pm 0.06 \mathrm{a} / \mathrm{x}$ & $\pm 0.22 \mathrm{a} / \mathrm{x}$ & $\pm 0.02 \mathrm{a} / \mathrm{y}$ & $\pm 0.14 \mathrm{a} / \mathrm{y}$ & $\pm 0.03 \mathrm{a} / \mathrm{z}$ & $\pm 0.17 \mathrm{a} / \mathrm{x}$ \\
& -1.53 & 6.38 & -1.72 & 8.71 & 0.06 & 6.21 \\
& $\pm 0.04 \mathrm{~b} / \mathrm{x}$ & $\pm 0.18 \mathrm{~b} / \mathrm{x}$ & $\pm 0.06 \mathrm{~b} / \mathrm{y}$ & $\pm 0.21 \mathrm{~b} / \mathrm{y}$ & $\pm 0.05 \mathrm{~b} / \mathrm{z}$ & $\pm 0.16 \mathrm{~b} / \mathrm{x}$
\end{tabular}

Different letters indicate significant differences $(p<0.05)$ between level of oil for each formulation (a, b, c) and between antioxidant type at the same level of oil (x, y, z). 
Table 3. Color and standard deviation of surimi gels fortified with menhaden oil and antioxidants addition: redness $\left(\mathrm{a}^{*}\right)$ and yellowness $\left(\mathrm{b}^{*}\right)$ at the beginning of storage time.

\begin{tabular}{|c|c|c|c|c|c|c|}
\hline \multirow{2}{*}{$\begin{array}{c}\text { Menhaden } \\
\text { oil }\end{array}$} & \multicolumn{2}{|c|}{ No antioxidant } & \multicolumn{2}{|c|}{ Rosemary } & \multicolumn{2}{|c|}{ Green tea } \\
\hline & $a^{*}$ & $b^{*}$ & $a^{*}$ & $\mathrm{~b}^{*}$ & $a^{*}$ & $\mathrm{~b}^{*}$ \\
\hline \multirow[t]{2}{*}{$1.5 \%$} & -1.82 & 6.06 & -2.11 & 8.31 & 0.06 & 6.21 \\
\hline & $\pm 0.04 \mathrm{a} / \mathrm{x}$ & $\pm 0.30 \mathrm{a} / \mathrm{x}$ & $\pm 0.04 \mathrm{a} / \mathrm{y}$ & $\pm 0.21 \mathrm{a} / \mathrm{y}$ & $\pm 0.06 \mathrm{a} / \mathrm{z}$ & $\pm 0.22 \mathrm{a} / \mathrm{x}$ \\
\hline \multirow[t]{2}{*}{$2.5 \%$} & -1.59 & 7.07 & -1.96 & 9.25 & 0.16 & 6.76 \\
\hline & $\pm 0.04 \mathrm{~b} / \mathrm{x}$ & $\pm 0.16 \mathrm{~b} / \mathrm{x}$ & $\pm 0.02 \mathrm{~b} / \mathrm{y}$ & $\pm 0.19 \mathrm{~b} / \mathrm{y}$ & $\pm 0.07 \mathrm{a} / \mathrm{z}$ & $\pm 0.15 \mathrm{~b} / \mathrm{z}$ \\
\hline
\end{tabular}

Different letters indicate significant differences $(\mathrm{p}<0.05)$ between level of oil for each 450 formulation (a, b, c) and between antioxidant type at the same level of oil (x, y, z). 
Figure 1. Changes in descriptive sensory attributes of surimi gels with no added oil. A -aroma, F flavor and AFT- aftertaste. Error bars represent the standard deviation of 6 panelist.

Figure 2. Changes in descriptive sensory attributes of surimi gels containing fish oil concentrate at a 455 level of $1.5 \%$ n-3 PUFA with and without antioxidants addition. A - aroma, F- flavor and AFT aftertaste. Error bars represent the standard deviation of 6 panelist.

Figure 3. Changes in descriptive sensory attributes of surimi gels containing fish oil concentrate at a level of $2.5 \%$ n-3 PUFA with and without the addition of antioxidants. A - aroma, F- flavor and AFT - aftertaste. Error bars represent the standard deviation of 6 panelist.

460 Figure 4. Changes in descriptive sensory attributes of surimi gels containing menhaden oil at a level of $1.5 \%$ n-3 PUFA with and without added rosemary and green tea. A- aroma, F-flavor, AFTaftertaste. Error bars represent the standard deviation of 6 panelist.

Figure 5. Changes in descriptive sensory attributes of surimi gels containing menhaden oil at a level of $2.5 \%$ n-3 PUFA with and without the addition of antioxidants. A - aroma, F- flavor and AFT 465 aftertaste. Error bars represent the standard deviation of 6 panelist.

Figure 6. Ratio of the relative polyene index (PI) of oils extracted from surimi gels to the PI of stock oil. PI was calculated as C22:6/C16:0. Clear bars represent gels before frozen storage; dark represent gels after 9 months of frozen storage.

Figure 7. Change in whiteness of surimi gels with oil and antioxidant addition. Error bars represent 470 the standard deviation of 6 samples.

Figure 8. Changes in shear stress of surimi gels with different oil and antioxidant addition. Error bars represent the standard deviation of 6 samples. 
Figure 9. Changes in shear strain of surimi gels with different oil and antioxidant addition. Error bars represent the standard deviation of 6 samples. 
Fish oil concentrate (1.5 \% n-3 PUFA)

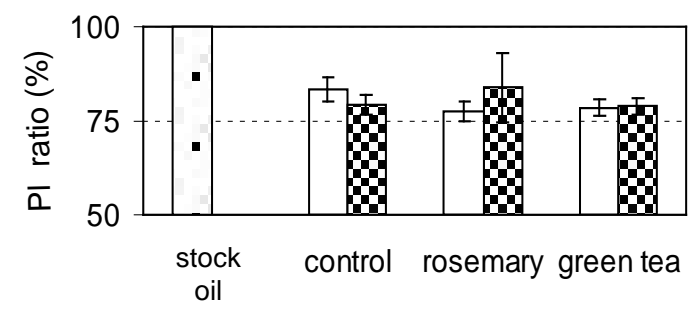

Fish oil concentrate (2.5\% n-3 PUFA)

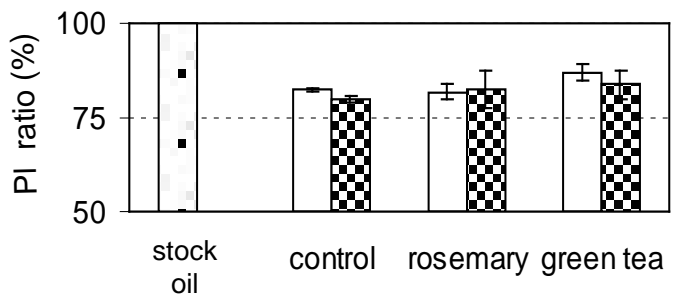

Menhaden oil (2.5 \% n-3 PUFA)

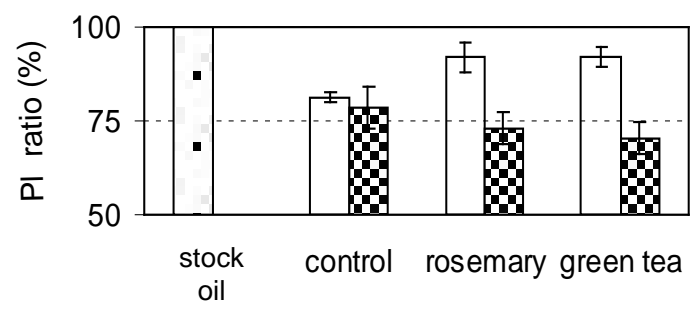

Menhaden oil (2.5 \% n-3 PUFA)

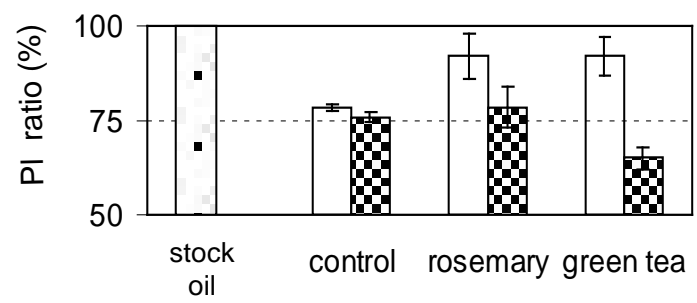

Figure 6 
Fish oil concentrate $(1.5 \% \mathrm{n}-3$ PUFA $)$

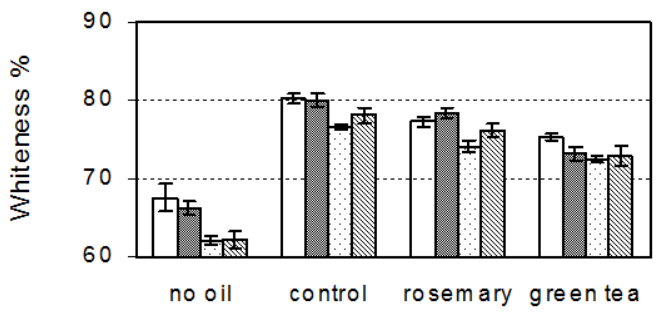

Fish oil concentrate $(2.5 \% \mathrm{n}-3$ PUFA)

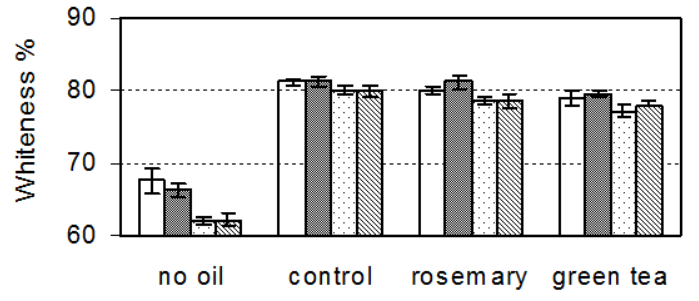

Menhaden oil (2.5\% n-3 PUFA)

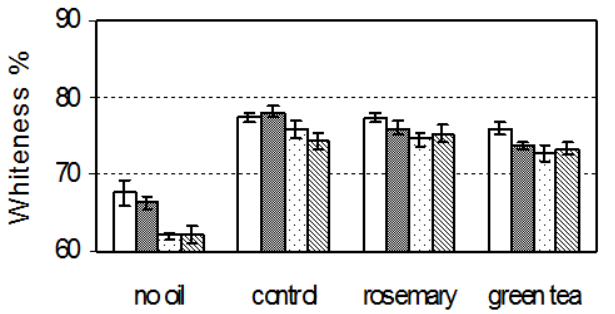

Menhaden oil (2.5 \% n-3 PUFA)

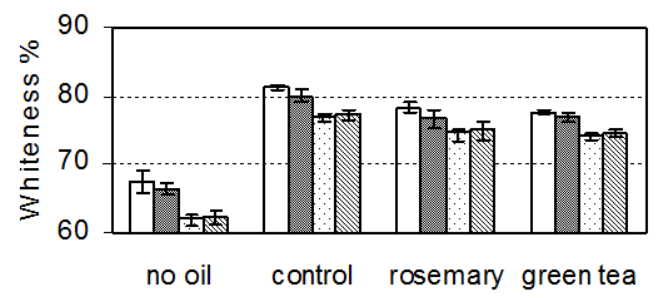

Figure 7 


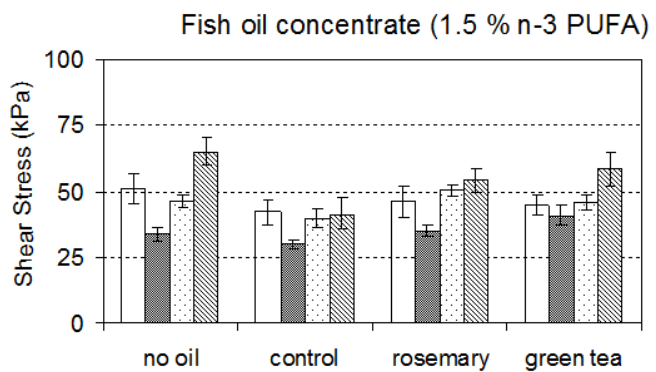

Fish oil concentrate $(2.5 \%$ n-3 PUFA)

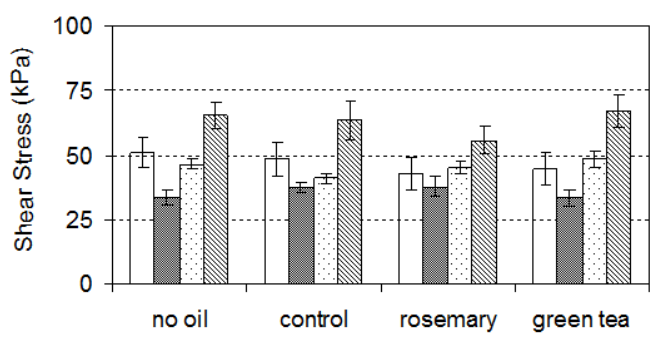

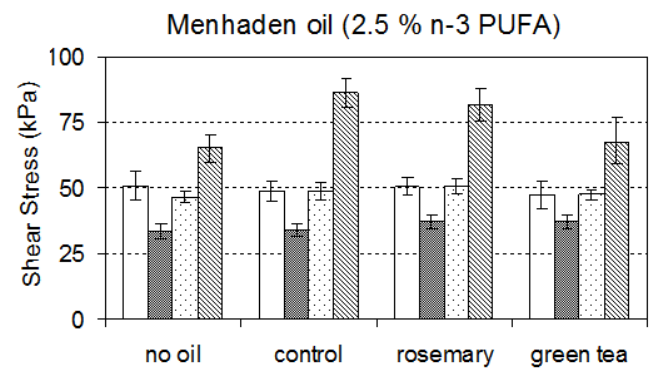

Menhaden oil (2.5 \% n-3 PUFA)

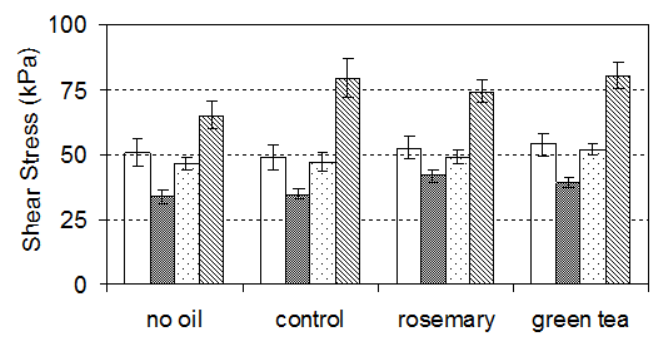

$\square 0 \square 3 \quad 6 \quad \mathbb{W} 9$ month

485

\section{Figure 8}



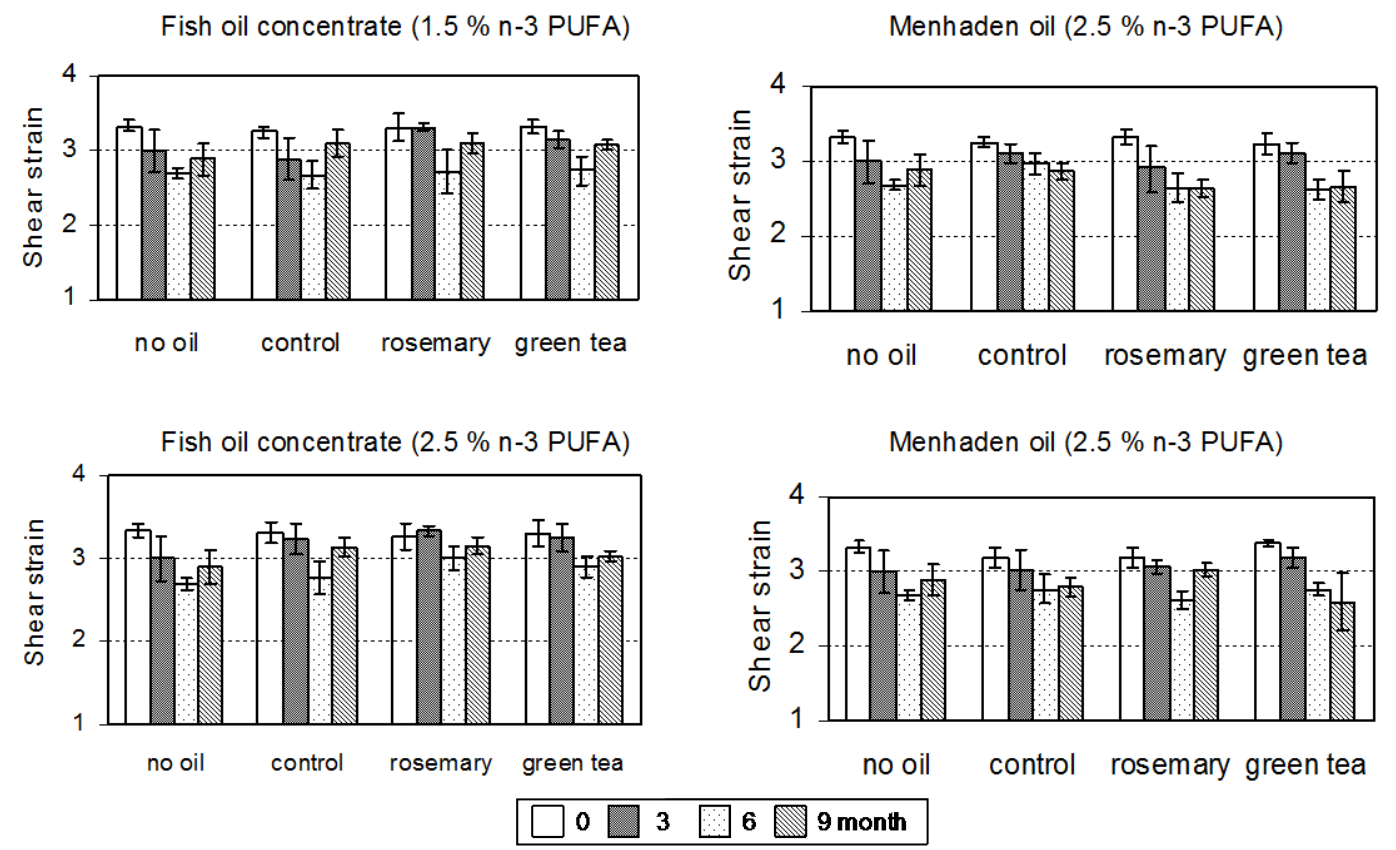

Figure 9. 